\title{
Las escorias de la central térmica GICC ELCOGAS como materia prima para la síntesis de materiales vitrocerámicos Parte 1: Comportamiento en fusión de las escorias GICC y obtención del vidrio original.
}

\author{
M. AINETO, A. ACOSTA. \\ Universidad de Castilla La Mancha, Facultad de Ciencias Químicas, Departamento de Química Física, Área de Mineralogía Aplicada. \\ C/ Camilo José Cela s/n 13071 Ciudad Real
}

\begin{abstract}
Se exponen aquí resultados de la primera parte de investigación sobre el comportamiento en fusión de las escorias de la central térmica GICC ELCOGAS y el proceso seguido para la obtención de un vidrio utilizando estas escorias como materia prima. Se ha diseñado una mezcla vitrificable (ECSCP) compuesta por un $40 \%$ de escorias, un $30 \%$ de casco de vidrio y un $30 \%$ de carbonato cálcico precipitado de azucarera, que permitió obtener por fusión a $1450{ }^{\circ} \mathrm{C}$ un vidrio homogéneo. El vidrio ECSCP ha sido caracterizado y se ha estudiado su cinética de cristalización a través de análisis térmicos que han puesto de manifiesto un mecanismo de cristalización preferente de tipo superficial. En una segunda parte de la investigación se utilizará este vidrio para obtener materiales vitrocerámicos de anortita/wolastonita.
\end{abstract}

\section{Palabras clave: Residuos de gasificación de carbón, escorias GICC, fusión, vitrificación, vitrocerámicos..}

The slag from ELCOGAS IGCC thermal power plant as raw material for the synthesis of glass-ceramic materials. Part I: Thermal behavior of the IGCC slag and synthesis of the parent glass.

We report here the results of the first phase of investigation on the melting behavior of the IGCC slag, and the use of this slag as raw component to produce glass ceramics. The vitrifying mixture named ECSCP, is composed by $40 \%$ of slag, $30 \%$ of glass cullet and $30 \%$ of precipitated calcium carbonate obtained as a by-product in a sugar refining plant. This mixture was melted at $1450^{\circ} \mathrm{C}$ to obtain the ECSCP parent glass, that was then characterized and its crystallization kinetics studied by thermal analysis. The ECSCP glass exhibit a surface mechanism of crystallization, and will be used to produce anorthite/ wollastonite glass ceramics in the second part of the investigation.

Keywords: Coal gasification wastes, IGCC slag, melting, vitrification, glassceramics.

\section{INTRODUCCIÓN}

Las centrales térmicas GICC (1) producen energía eléctrica mediante una tecnología diferente de la utilizada en las centrales térmicas convencionales. El proceso GICC consta de una primera etapa, la gasificación del combustible, seguida de un ciclo combinado convencional. La central GICC ELCOGAS, S.A. (2) situada en Puertollano (Ciudad Real) con una potencia de $320 \mathrm{MW}$, emplea como combustible una mezcla al 50\% de carbón y coque de petróleo. El volumen de residuos generado en esta central es de 100.000 toneladas / año, aproximadamente un $85 \%$ de escorias y un $15 \%$ de cenizas volantes. Se ha probado la idoneidad de las cenizas y escorias como material puzolánico para fabricar cementos y hormigones (3) y como aditivo en la fabricación de ladrillos cerámicos (4), (5).

Las escorias de la central térmica ELCOGAS (6) son el material objeto de esta investigación. Son generadas durante la gasificación del combustible, proceso en el cual, la parte mineral no gasificable del carbón funde por efecto de las altas temperaturas en condiciones reductoras y alta presión gaseosa. El fundido es enfriado bruscamente por inmersión en agua y solidifica en forma de escorias, un sólido granular vítreo de color negro brillante con un tamaño de partícula similar a una arena.
Un campo de aplicación en el que el uso de estas escorias puede representar una ventaja respecto a otras materias primas es el de los materiales vitrocerámicos $(7,8)$ pues su fusión tiene lugar a temperaturas más bajas que una mezcla mineral de la misma composición. La utilización de las escorias en este campo, además de las obvias ventajas medioambientales derivadas del aprovechamiento de residuos, puede suponer también un ahorro económico al sustituir, sin gastos de extracción ni procesado previo, a otras materias primas minerales de mayor valor añadido.

El aprovechamiento de diversos tipos de residuos para producción de materiales vítreos está ampliamente documentado, con trabajos de revisión que reconocen el interés económico y ecológico de esta solución para el reciclado de residuos $(9,10,11,12)$. Sin embargo, no se conocen trabajos de esta índole relativos a residuos de centrales térmicas de gasificación, debido a que se trata de un material de producción relativamente reciente.

El objetivo de este trabajo es el estudio del comportamiento en fusión de las escorias de gasificación de carbón y la obtención y caracterización de un vidrio original basado en las escorias utilizando como fundentes otros materiales 
también residuales de fácil disponibilidad en un entorno cercano al lugar de producción. Este vidrio será utilizado en una segunda parte de la investigación para obtener materiales vitrocerámicos.

\section{MATERIALES Y METODOLOGÍA DE ANÁLISIS}

La composición química de las escorias GICC, similar a la de las cenizas del carbón, es básicamente sílico-aluminosa $(>80 \%)$, con contenidos menores de óxidos de hierro, calcio, magnesio y alcalinos (Tabla I). Otros materiales empleados (Tabla II) son el casco de vidrio reciclado (CS) y el carbonato precipitado $(\mathrm{CP})$ procedente de la azucarera EBRO Agrícolas de Ciudad Real (13). Este material se genera como subproducto en el proceso de depuración de la remolacha. Se trata de un lodo que una vez seco se encuentra en forma de polvo compactado, con una riqueza en carbonato cálcico en torno al $95 \%$.
Los análisis por microscopía de calefacción se han realizado en un equipo Misura ensayando las muestras con un tamaño de partícula fino $\left(\mathrm{d}_{50}<20 \mu \mathrm{m}\right)$, sobre soporte de alúmina a velocidad de calentamiento de $5^{\circ} \mathrm{C} / \mathrm{min}$, en atmósfera al aire, condiciones que permiten una correlación entre la temperatura a la que se observa una determinada forma geométrica y su viscosidad correspondiente (14).

\section{PARTE EXPERIMENTAL}

En primer lugar se abordó el estudio del comportamiento en fusión de las escorias GICC. El análisis térmico diferencial y termo-gravimétrico se registró a una velocidad de calentamiento de $25^{\circ} \mathrm{C} / \mathrm{min}$ sobre escorias en polvo. La prueba de fusión de escoria sin adiciones se realizó en horno eléctrico a $1450^{\circ} \mathrm{C}$, manteniendo esa temperatura durante 1 hora. El fundido no se pudo colar dada su elevada viscosidad y la acusada tendencia a formar burbujas.

TABLA I. ESCORIAS (ES): COMPOSICIÓN QUÍMICA (\% PESO)

\begin{tabular}{|c|c|c|c|c|c|c|c|c|c|c|}
\hline $\begin{array}{c}\mathrm{SiO}_{2} \\
(\mathrm{XRF})\end{array}$ & $\mathrm{Al}_{2} \mathrm{O}_{3}(\mathrm{XRF})$ & $\mathrm{FeO}$ (TITR) & $\mathrm{MnO}(\mathrm{XRF})$ & $\mathrm{MgO}(\mathrm{XRF})$ & $\mathrm{CaO}(\mathrm{XRF})$ & $\mathrm{Na}_{2} \mathrm{O}(\mathrm{XRF})$ & $\mathrm{K}_{2} \mathrm{O}(\mathrm{XRF})$ & $\mathrm{TiO}_{2}(\mathrm{XRF})$ & $\begin{array}{c}\mathrm{C} \\
\text { (IR) }\end{array}$ & $\begin{array}{c}\mathrm{S} \\
\text { (IR) }\end{array}$ \\
\hline 55.49 & 28.39 & 5.81 & 0.05 & 0.94 & 6.08 & 0.4 & 2.4 & 0.77 & 0.38 & 0.51 \\
\hline
\end{tabular}

TABLA II. COMPOSICIÓN QUÍMICA (FRX) CP: CARBONATO PRECIPITADO DE AZUCARERA; CS: CASCO DE VIDRIO; ECSCP: VIDRIO.

\begin{tabular}{|c|c|c|c|c|c|c|c|c|c|c|c|c|}
\hline & $\mathrm{SiO}_{2}$ & $\mathrm{Al}_{2} \mathrm{O}_{3}$ & $\mathrm{Fe}_{2} \mathrm{O}_{3}$ & $\mathrm{MnO}$ & $\mathrm{MgO}$ & $\mathrm{CaO}$ & $\mathrm{Na}_{2} \mathrm{O}$ & $\mathrm{K}_{2} \mathrm{O}$ & $\mathrm{TiO}_{2}$ & $\mathrm{P}_{2} \mathrm{O}_{5}$ & PPC & Suma \\
\hline $\mathrm{CP}$ & 2.25 & 0.82 & 0.33 & 0.02 & 1.32 & 47.37 & 0.05 & 0.25 & 0.05 & 1.22 & 44.76 & 98.42 \\
\hline CS & 72.47 & 1.77 & 1.19 & n.d. & 1.30 & 10.89 & 12.38 & 0.84 & 0.080 & n.d. & n.d. & 100.92 \\
\hline ECSCP & 53.14 & 14.40 & 3.17 & 0.06 & 1.63 & 20.56 & 5.00 & 1.40 & 0.43 & 0.40 & n.d. & 100.19 \\
\hline
\end{tabular}

El desarrollo de esta investigación se ha apoyado en una serie de técnicas analíticas necesarias para caracterización físico-química, mineralógica y térmica tanto de las materias primas de partida, como del vidrio sintetizado. El análisis químico de las escorias y del carbonato cálcico precipitado de azucarera se llevó a cabo en los laboratorios ACTLABS de Canadá, especializados en análisis Geoquímicos. Los elementos mayoritarios se analizaron por fluorescencia de rayos $\mathrm{X}$ en perla fundida, el contenido en carbono y azufre total por espectroscopia infrarroja y la concentración de oxido ferroso se determinó por análisis titrimétrico.

La composición química del casco de vidrio y del vidrio obtenido tras la fusión se ha llevado a cabo por fluorescencia de rayos X en un equipo PHILIPS Magix Pro. El ensayo se hizo en perla fundida a $1100^{\circ} \mathrm{C}$ preparada con $0,5 \mathrm{~g}$ de muestra y $5 \mathrm{~g}$ de tetraborato de litio. Los resultados se interpretaron a través de un programa IQ+ en base a una serie de patrones de muestras minerales genéricas. Los análisis mineralógicos por difracción de rayos $X$ se han realizado en un difractómetro PHILIPS PW-1710 con rendija automática de divergencia y monocromador de grafito empleando la radiación $\mathrm{K} \alpha$ del cobre. El ensayo se realizó compactando en un porta de aluminio las muestras secadas a $110^{\circ} \mathrm{C}$ y pulverizadas a tamaños de partícula por debajo de 50 micras. Los análisis térmico-diferencial y termogravimétricos (ATD/TG) se han llevado a cabo en un equipo Setaram Labsys, en atmósfera aire, con crisoles de platino y estándar de referencia de alúmina calcinada en polvo. Las escorias se han ensayado también en atmósfera de argón.
La mezcla ECSCP compuesta por un $40 \%$ de escorias, un $30 \%$ de casco de vidrio y un $30 \%$ de carbonato cálcico precipitado de azucarera tiene una composición, situada en torno al eutéctico de $1170^{\circ} \mathrm{C}$ del diagrama de fases $\mathrm{CaO}-\mathrm{Al}_{2} \mathrm{O}_{3}$ $\mathrm{SiO}_{2}$. Esta mezcla, con bajo punto de fusión, permitió obtener un fundido de menor viscosidad.

La fusión se llevó a cabo en un crisol sílico-aluminoso calentado en horno eléctrico a una temperatura de $1450^{\circ} \mathrm{C}$ durante 1 hora. El fundido fue colado del sobre un molde de latón dando lugar a un vidrio homogéneo de color negro que fue inmediatamente recocido a $550^{\circ} \mathrm{C}$ durante 2 horas. El vidrio se caracterizó analizándose su composición química, el espectro de rayos $X$, sus propiedades térmicas y su mecanismo y cinética de cristalización a través de ensayos térmicos en diferentes tamaños de partícula y velocidades de calentamiento.

\section{RESULTADOS Y DISCUSIÓN}

\section{Comportamiento térmico y en fusión de las escorias GICC}

$\mathrm{El}$ gráfico de ATD de las escorias a $10^{\circ} \mathrm{C} / \mathrm{min}$ en aire (Fig. 1) muestra una señal exotérmica a $685^{\circ} \mathrm{C}$ debida a la oxidación del hierro II a hematita (15) y a restos de combustible sin quemar; otro exotérmico a $1055^{\circ} \mathrm{C}$ es debido a la cristalización de mullita, como se pone de manifiesto en los gráficos de difracción de rayos $X$ de la Fig 2, donde se pueden apreciar los picos de hematita desarrollados durante el tratamiento 


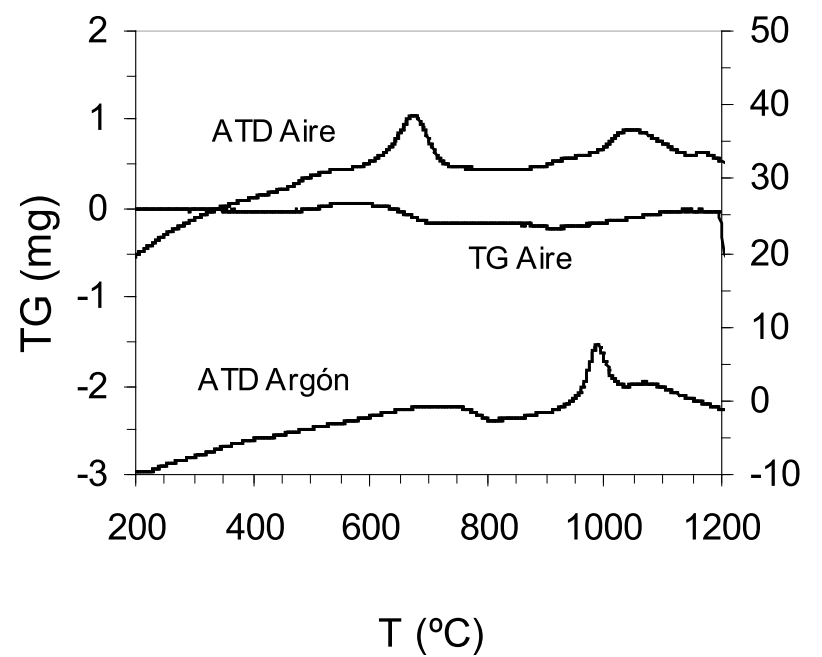

Fig. 1.- Gráficos de ATD-TG de las escorias GICC.

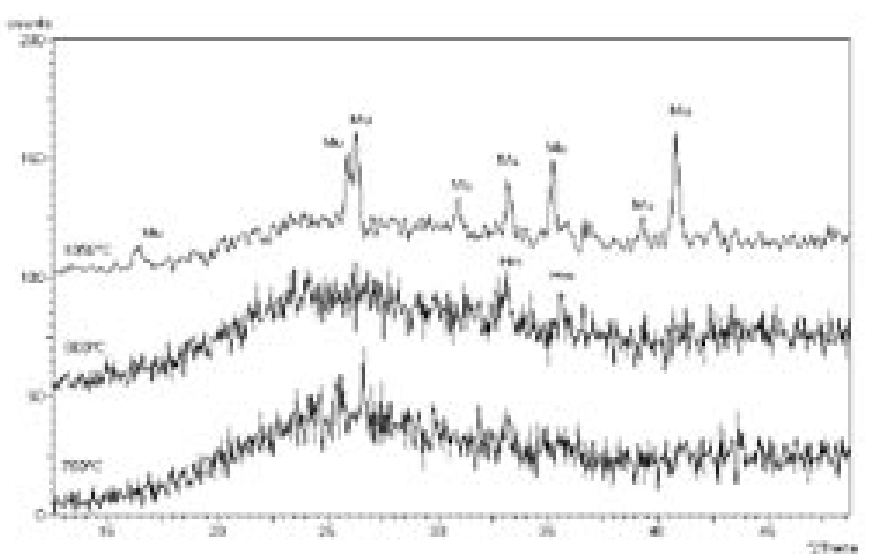

Fig. 2.- Diafractogramas de las escorias GICC después de ser tratadas a $750^{\circ} \mathrm{C}, 4$ horas; $850^{\circ} \mathrm{C} 4$ horas y $1050^{\circ} \mathrm{C}, 15$ minutos. Mu: Mullita, $\mathrm{Hm}$ : Hematita.

isotermo a $850^{\circ} \mathrm{C}$ durante un tiempo de 4 horas y de mullita en el tratamiento a $1050^{\circ} \mathrm{C}$ durante 15 minutos. Los picos de hematita detectados a temperaturas más bajas no se distinguen bien en los gráficos.

La temperatura de transición vítrea $\mathrm{Tg}$ no se puede determinar con precisión en el análisis en aire, por la simultánea concurrencia de los exotérmicos provocados por las reacciones de oxidación. El análisis de ATD de las escorias llevado a cabo en atmósfera de argón permite determinar este parámetro a $780^{\circ} \mathrm{C}$. La pérdida total de peso detectada en el análisis termogravimétrico está en torno a un 0,5\%, observándose oscilaciones que bien pueden ser errores ocurridos en el proceso de toma de datos del aparato, o pequeños incrementos por las oxidaciones. La prueba de fusión llevada a cabo a $1450^{\circ} \mathrm{C}$ da lugar a un fundido muy viscoso con gran cantidad de burbujas ocluidas en su interior, que no pudo ser colado. Esta tendencia a la desgasificación de las escorias y la formación de burbujas se puede observar también por el efecto expansivo a temperaturas por encima de $1300{ }^{\circ} \mathrm{C}$ que queda registrado en la curva de sinterización (Fig. 3A) obtenida por microscopía de calefacción.

\section{Caracterización de vidrio de origen ECSCP}

Se comprobó la composición química del vidrio ECSCP (Tabla II) y su espectro de difracción de rayos $\mathrm{X}$ sin picos,

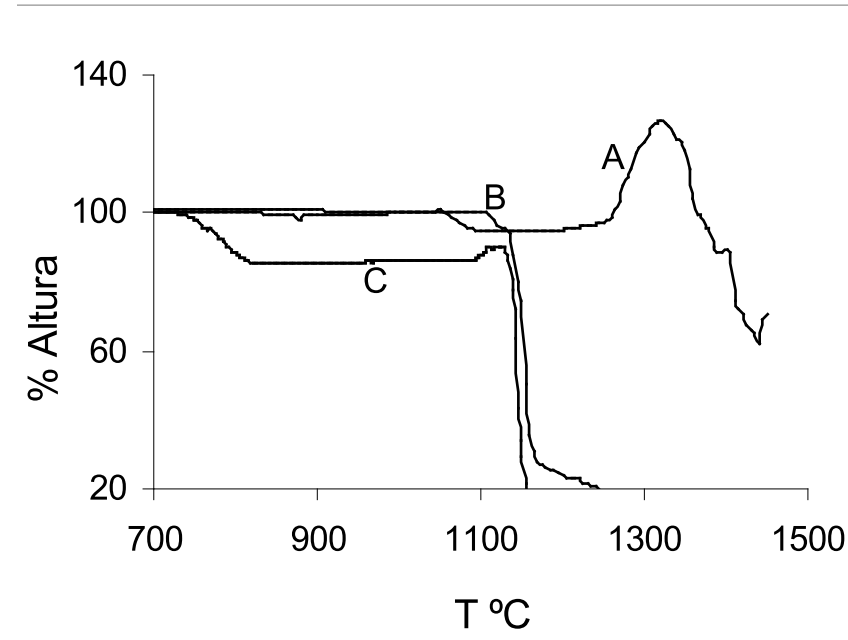

Fig. 3.- Gráfico de sinterización de las escorias GICC (A), la mezcla ECSCP en crudo (B) y el vidrio ECSCP (C).

característico de los materiales vítreos (Fig.4). El análisis por microscopía de calefacción determina una temperatura de fusión de $1150^{\circ} \mathrm{C}$ y muestra la desaparición del efecto expansivo observado en las escorias (Fig. 3C). El vidrio ECSCP es del tipo "corto", es decir, su evolución desde el punto de reblandecimiento a la fusión se da en un margen muy corto de temperaturas. Se trata de un vidrio con escasa tendencia a desvitrificar durante su enfriamiento, como se demuestra por la ausencia de picos de cristalización en la parte descendente de las curvas de ATD de enfriamiento a velocidades gradualmente lentas de 20,10 y $7^{\circ} \mathrm{C} / \mathrm{min}$ (Fig. 5).

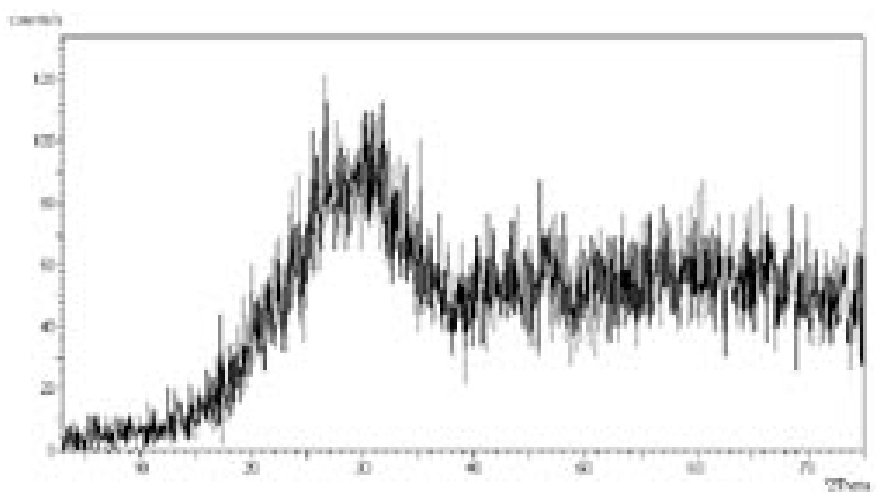

Fig. 4.- Difractograma del vidrio ECSCP

$\mu \mathrm{V}$

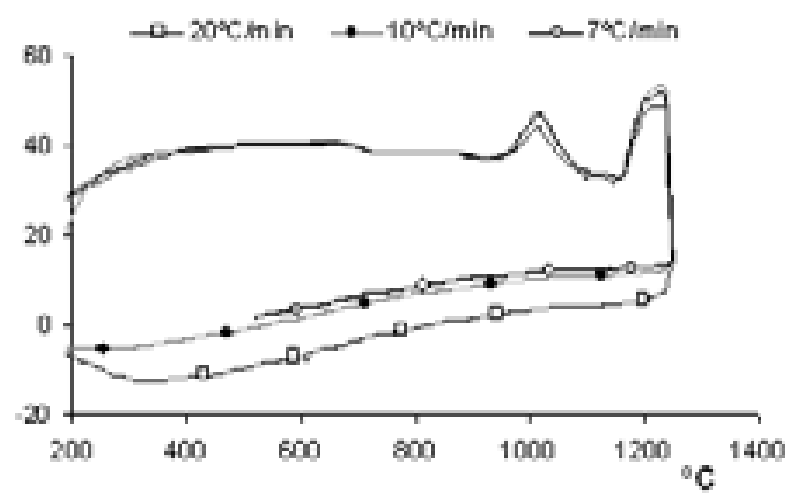

Fig. 5.- Enfriamiento a velocidades decrecientes del vidrio ECSCP 


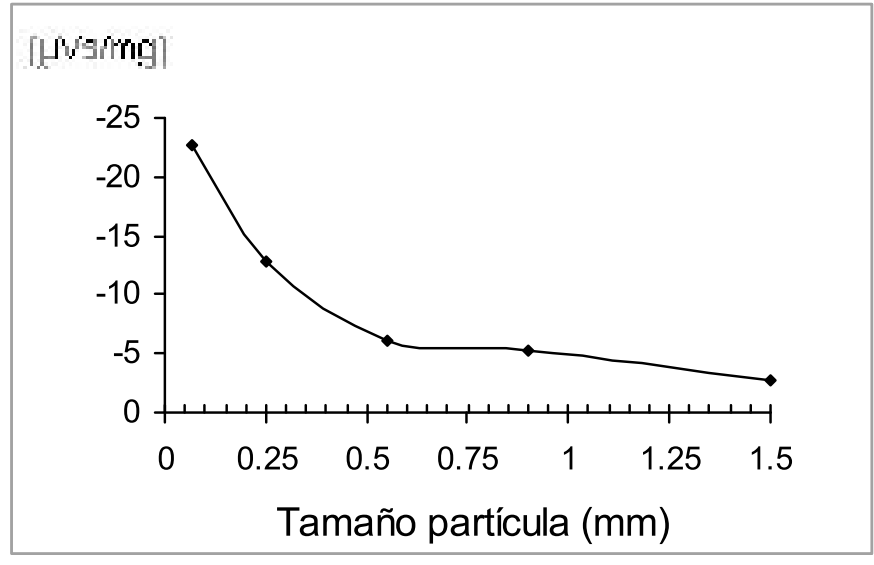

Fig. 6.- Área del pico de cristalización del vidrio ECSCP en función del tamaño de la partícula.

TABLA III.

\begin{tabular}{|c|c|c|}
\hline $\begin{array}{c}\text { INTERVALO DE TAMAÑOS } \\
\text { DE PARTíCULA } \\
\mathrm{mm}\end{array}$ & $\begin{array}{c}\text { VALOR MEDIO } \\
\text { INTERVALO } \\
\mathrm{mm}\end{array}$ & $\begin{array}{c}\text { ÁREA PICO } \\
\text { CRISTALIZACIÓN } \\
\mu \mathrm{Vs} / \mathrm{mg}\end{array}$ \\
\hline $1,25-1,70$ & 1.5 & -2.8 \\
\hline $0.8-1.0$ & 0.9 & -5.3 \\
\hline $0.5-0.6$ & 0.55 & -6 \\
\hline $0.2-0.3$ & 0.25 & -12.9 \\
\hline $0.063-0.080$ & 0.07 & -22.7 \\
\hline Todo uno $<0.160$ & & -22.8 \\
\hline
\end{tabular}

El mecanismo de cristalización preferente que presenta este vidrio se estudió mediante análisis térmico diferencial a $10^{\circ} \mathrm{C} / \mathrm{min}$ sobre las distintas fracciones granulométricas que se detallan en la Tabla 3. Se calculó el área del pico de cristalización obtenido en cada una de las curvas de ATD y se representó la curva de la Fig 6 en la que puede observarse una disminución de la entalpía de cristalización al aumentar el tamaño de partícula, lo que demuestra que este vidrio presenta un mecanismo de cristalización preferente de tipo

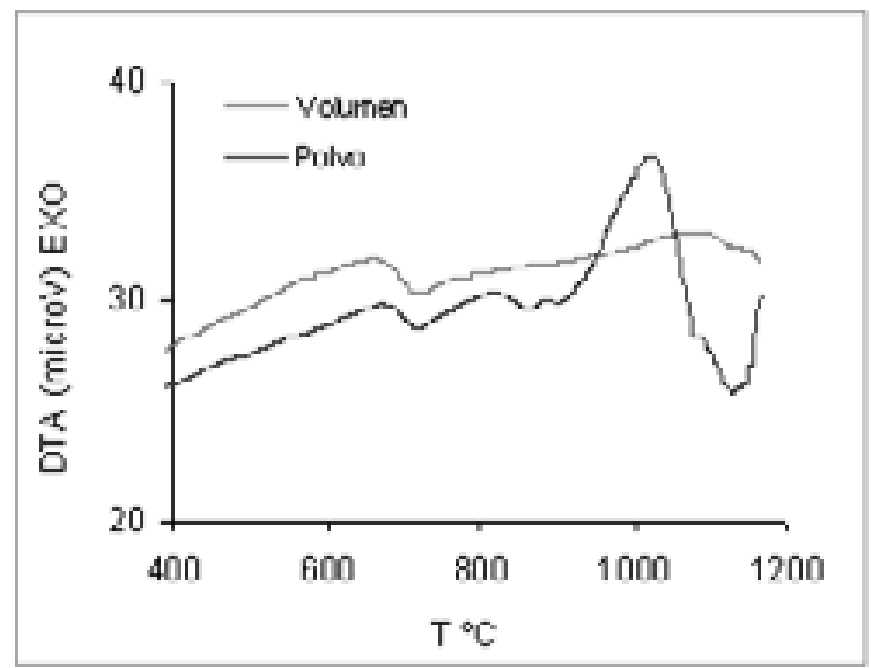

Fig. 7.- ATD-TG del vidrio ECSCP sobre muestras en polvo y volumen
TABLA IV. DATOS DE VELOCIDAD DE CALENTAMIENTO Y TEMPERATURA DEL MÁXIMO DEL PICO DE CRISTALIZACIÓN PARA EL CÁLCULO DE LA ECUACIÓN DE KISSINGER.

\begin{tabular}{|c|c|c|c|c|}
\hline $\begin{array}{c}\phi \\
{ }^{\circ} \mathrm{C} / \mathrm{min}\end{array}$ & $\begin{array}{c}\mathrm{Tp} \\
{ }^{\circ} \mathrm{C}\end{array}$ & $\begin{array}{c}\mathrm{Tp} \\
{ }^{\circ} \mathrm{K}\end{array}$ & $\begin{array}{c}1 / \mathrm{Tp} \\
{ }^{\circ} \mathrm{K}^{-1}\end{array}$ & $\begin{array}{c}\ln \left(\phi / \mathrm{Tp}^{2}\right) \\
{ }^{\circ} \mathrm{K}^{-1} \mathrm{~min}^{-1}\end{array}$ \\
\hline 10 & 954.5 & 1227.5 & 0.81 & -11.92 \\
\hline 20 & 982.6 & 1255.6 & 0.80 & -11.28 \\
\hline 30 & 1000.6 & 1273.6 & 0.79 & -10.90 \\
\hline 40 & 1010.4 & 1283.4 & 0.78 & -10.63 \\
\hline 50 & 1024 & 1297 & 0.77 & -10.42 \\
\hline
\end{tabular}

superficial, como se deduce de las curvas de la Fig. 7, donde la muestra en polvo presenta un pico de cristalización en torno a $1000^{\circ} \mathrm{C}$ mientras que en la muestra en volumen, el pico de cristalización es apenas perceptible.

Los análisis de ATD sobre el vidrio en polvo de tamaño de partícula menor de $160 \mu \mathrm{m}$ a velocidades de calentamiento de $10,20,30,40$ y $50{ }^{\circ} \mathrm{C} / \mathrm{min}$ han permitido obtener los datos de la Tabla IV, que permiten trazar la recta que relaciona la velocidad de calentamiento $\phi$ con la temperatura a la que aparece el máximo del pico de cristalización Tp y que corresponde a la ecuación de Kissinger (16):

$$
\ln \frac{\phi}{T_{p}^{2}}=-\frac{E_{a}}{R T_{p}}+c t e
$$

De la pendiente de la recta (Fig. 8), se deducen los parámetros cinéticos de cristalización del vidrio: la energía de activación $(\mathrm{Ea}=299 \mathrm{KJ} / \mathrm{mol})$ y el coeficiente $\mathrm{n}$ de Avrami dado por la expresión:

$$
n=\frac{2.5}{\Delta T} x \frac{R T_{p}^{2}}{E_{a}}
$$

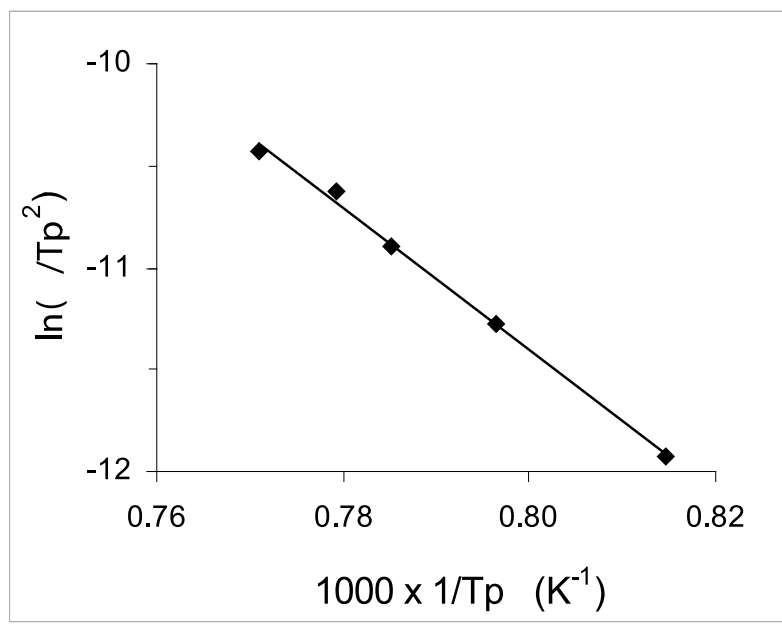

Fig. 8.- Representación de Kissinger: $\phi$ velocidad de calentamiento. Tp: Temperatura del máximo del pico de cristalización. 
Donde $\Delta T$ es la anchura a la semialtura del pico de cristalización y R la constante de los gases. El valor medio del coeficiente de Avrami calculado para los cinco picos de cristalización resultantes de los ensayos a las velocidades anteriormente mencionadas, es de 2.6.

En la Tabla V se resumen los resultados de los distintos análisis térmicos efectuados sobre el vidrio ECSCP.

La viscosidad del vidrio se ha calculado a través de tres pares de valores de viscosidad/temperatura obtenidos en los ensayos de ATD y de microscopía de calefacción del vidrio. Estos puntos fijos de viscosidad vienen definidos por la forma geométrica que adquiere una probeta fabricada con polvo de la muestra vítrea a medida que la temperatura asciende. Con estos pares de valores se calculan las constantes A, B y To de la ecuación de Vogel-Fulcher- Tamman (17):

$$
\log =A+\frac{B}{T-T_{0}}
$$

La ecuación de viscosidad del vidrio ECSCP fue obtenida a partir de los puntos de temperatura de transición vítrea $\left(708^{\circ}\right.$ C) obtenido en el ensayo de ATD a $10^{\circ} \mathrm{C} / \mathrm{min}$, y los puntos de máxima contracción $\left(818^{\circ} \mathrm{C}\right)$ y fusión $\left(1156^{\circ} \mathrm{C}\right)$ obtenidos

TABLA V. PARÁMETROS DEL VIDRIO ECSCP OBTENIDOS POR ATD.

\begin{tabular}{|c|c|c|c|c|c|}
\hline $\begin{array}{c}\text { Transición } \\
\text { vítrea Tg }\end{array}$ & $\begin{array}{c}\text { Cristalización } \\
\mathrm{Tp} \\
{ }^{\circ} \mathrm{C}\end{array}$ & $\begin{array}{c}\text { Fusión } \\
\mathrm{Tm}\end{array}$ & $\begin{array}{c}\text { Energía de } \\
\text { Activación }\end{array}$ & $\begin{array}{c}\text { Coeficiente } \\
\text { de Avrami }\end{array}$ & $\begin{array}{c}\text { Tipo de } \\
\text { cristalización } \\
\text { preferente }\end{array}$ \\
\hline 708 & 829 & 1156 & 299 & 2.6 & KJ/mol \\
\hline 1031 & & & & \\
\hline
\end{tabular}

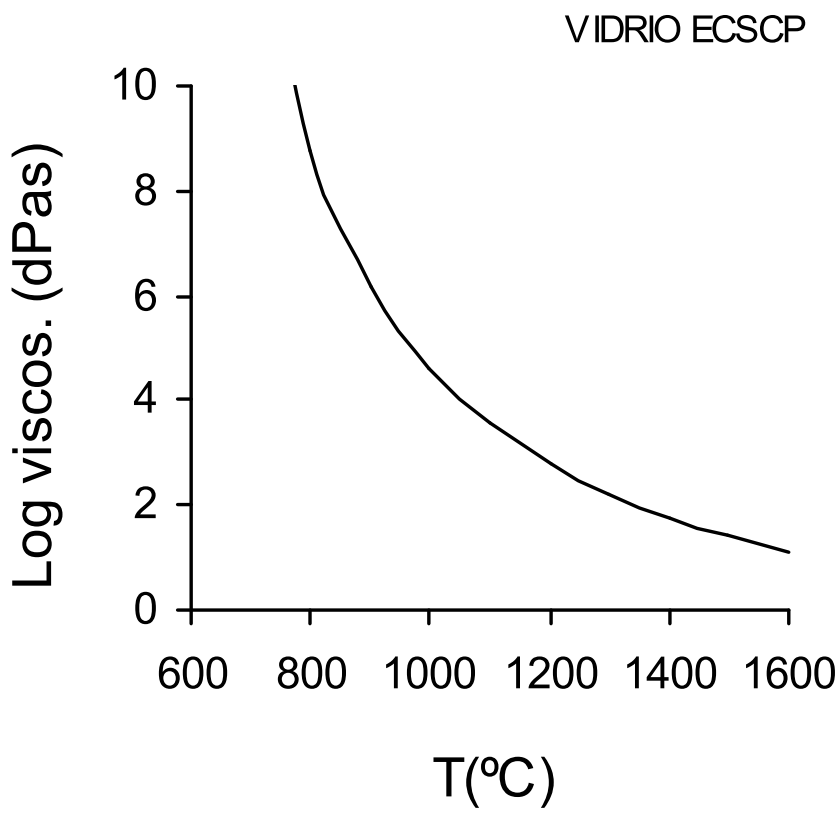

Fig. 9.- Curva de viscosidad del vidrio ECSCP. por microscopía de calefacción. En las condiciones en que se llevaron a cabo los análisis es posible correlacionar estos puntos con valores de viscosidad de $10^{13.3}, 10^{8.2}$ y $10^{3.1}$. Las constantes A, B y To calculadas a partir de estos pares de valores son respectivamente -1.82 , 3268 y 492; quedando la curva de viscosidad (Fig. 9) del vidrio ECSCP definida por la expresión:

$$
\log \eta \underset{\eta}{=}-1.82+\frac{3268}{T-492}
$$

Estos valores de las constantes de la ecuación de VogelFulcher-Tamman obtenidos están en el mismo rango de los descritos para vidrios de composiciones similares $(14,18)$.

\section{CONCLUSIONES}

El tratamiento térmico para la fusión de las escorias GICC provoca un proceso de desgasificación con desprendimiento de una gran cantidad de burbujas procedentes de la liberación de los gases que estaban disueltos en la matriz vítrea constitutiva de este residuo. Considerando que la composición de las escorias GICC presentan un contenidos en sílice y alúmina por encima del $80 \%$, el fundido resultante de su calentamiento a $1450^{\circ} \mathrm{C}$ presenta una alta viscosidad. Los gases desprendidos encuentran grandes dificultades para ascender a la superficie originado el espumado que se observa durante su fusión. Este fundido viscoso y espumado fluye mal en el proceso de colado impidiendo la obtención de un vidrio homogéneo. Este efecto se aprecia claramente en los análisis por microscopía de calefacción, por el efecto expansivo característico de la curva de sinterización.

Se hace imprescindible por tanto, mezclar estas escorias con otros materiales que aporten óxidos modificadores y fundentes, de modo que se rebaje la proporción de sílice y alúmina en su composición y disminuya la viscosidad del fundido para favorecer la liberación de estos gases. La mezcla basada en un $40 \%$ de escorias, $30 \%$ de casco de vidrio y $30 \%$ de carbonato precipitado residual de azucarera, de composición eutéctica en el diagrama de fases $\mathrm{CaO} \mathrm{SiO}_{2} \mathrm{Al}_{2} \mathrm{O}_{3^{\prime}}$, da lugar a un vidrio homogéneo con buena fluencia en el proceso de colado, que puede ser utilizado posteriormente para obtención de materiales vitrocerámicos basados en el sistema anortita/wolastonita.

Se han obtenido los parámetros cinéticos del vidrio resultando una energía de activación del proceso de cristalización de $299 \mathrm{KJ} / \mathrm{mol}$ y un coeficiente de Avrami de 2.6 correspondiente a un crecimiento cristalino tridimensional. Este vidrio, presenta un mecanismo superficial de cristalización, por lo que su desvitrificación se verá favorecida si se lleva a cabo a por el procedimiento de sinterización-cristalización del vidrio en polvo prensado.

\section{AGRADECIMIENTOS}

Las siguientes entidades han colaborado en la financiación de esta investigación: la Comisión Interministerial de Ciencia y Tecnología a través del proyecto REN2001-0912/TECNO; las empresas ELCOGAS S.A y Azucarera EBRO Agrícolas. 


\section{BIBLIOGRAFÍA}

1. M. Triviño, Tecnología de gasificación integrada en ciclo combinado Ed ELCOGAS, S.A. y Club Español de la Energía, Madrid, (España) 2003. (http:/ / w.w.w. elcogas.es)

2. R. Delgado Taberner, "La central GICC de Puertollano. Producción limpia de electricidad mediante la gasificación del carbón", Las centrales eléctricas de ciclo combinado. 2001. (http:/ / www.aepm.org)

3. A. Acosta, M. Aineto, I. Iglesias, S. Burgos P. Puente: "Valorisation of IGCC by-products from the Puertollano Power Plant. Proceedings of International Workshop on Novel Products from Combustion Residues: Opportunities and Limitations Morella, Spain, June 2001.

4. A. Acosta, I. Iglesias, M. Aineto, M. Romero and J. Ma. Rincón, “Utilisation of IGCC slag and clay steriles in soft mud bricks for use in building bricks manufacturing". Waste Management, 22 (8) 887-891 (2002).

5. M. Aineto, I. Iglesias, A. Acosta, "Gasification fly ash in building ceramics". Global Symposium on Recycling, Waste Treatment and Clean Technology, REWAS 2004, Madrid, (España) 2004.

6. A. Acosta, M. Aineto, I. Iglesias: “Physico-Chemical characterization of slag waste coming from IGCC Thermal Power Plant". Mater. Lett., 50, 246-250 (2001).

7. I. De Vicente-Mingarro, P. Callejas, J.M ${ }^{a}$ Rincon,: "Materiales vitroceramicos: el proceso vitroceramico Bol. Soc. Esp. Ceram. V., 1993, 32 ( 3) 157- 167.

8. Z. Strnad, Glass-ceramic materials 1986. Elsevier, Amsterdam.
9. A.V. Gorokhovskii, V.A. Gorokhovskii, D.V. Meshcheryakov, A.A Kopchekchi, "Production of Glass Ceramics as a Method for Comprehensive Utilization of Chemical Industry Waste", Glass Ceram+., 59 (9-10), 353-355 (2002).

10 M. Romero, J.M ${ }^{\mathrm{a}}$ Rincón,: “El proceso de vitrificación/cristalización controlada aplicado al reciclado de residuos industriales inorgánicos". Bol. Soc. Esp. Ceram. V. 2000, 39 ( 1) 155- 163.

11. L. Barbieri, A. Corradi, I. Lancellotti "Waste based glasses and glass ceramics" Mater. Construcc. 2001, 5, (263-264) 197-208.

12. L Barbieri, I. Lancellotti, T. Manfredini, I. Queralt, J. Ma Rincón, M. Romero, "Design, obtainment and properties of glasses and glass-ceramics from coal fly ash" Fue, 1999 78, 271-276.

13. A. Acosta, M. Aineto, M. Ruiz Holst, A. García Murillo, R. Pozo, Sugar precipitated calcium carbonate properties en III Congress of Added Value and Recycling of Industrial Waste" L'Aquila, (Italia) 2001.

14. M. J. Pascual, L. Pascual, A. Durán,: Determination of the viscositytemperature curve for glasses on the basis of fixed viscosity points determined by hot stage microscopy Phys. Chem. of Glasses, 42, (1), 61-66 (2001).

15. A. Acosta, I. Iglesias, M. Aineto: “Thermal and sintering characterization of IGCC Slag". J. Therm. Anal. Calorim. 2002, 67, 249-255.

16. J. Malek, "Crystallization Kinetics by Thermal Analysis," J. Therm. Anal. Calorim. 1999, 56, 763-769.

17. J.M. Fernández Navarro: “Viscosidad" pp. 337-372, en El Vidrio, Consejo Superior de Investigaciones Científicas, Fundación Centro Nacional del Vidrio, Madrid (España) 1991.

18. A. De Pablos, A.Durán, M. I. Nieto, "Puesta a punto de un horno de fibrado de laboratorio para la obtención de fibra de vidrio" Bol. Soc. Esp. Ceram. V.. 1997, 36 ( 5) 517- 524.

Recibido: 10.01 .05

Aceptado: 17.06 .05 